\title{
Stress-strength Reliability of Exponential Distribution based on Type-I Progressively Hybrid Censored Samples
}

\author{
S. M. Mirjalili, H. Torabi, H. Nadeb* and S. F. Bafekri \\ Yazd University
}

Received: 2/5/2016 Approved: 10/16/2016

\begin{abstract}
This paper considers the estimation of the stress-strength parameter, say $R$, based on two independent Type-I progressively hybrid censored samples from exponential populations with different parameters. The maximum likelihood estimator and asymptotic confidence interval for $R$ are obtained. Bayes estimator of $R$ is also derived under the assumption of independent gamma priors. A Monte Carlo simulation study is used to evaluate the performance of maximum likelihood estimator, Bayes estimator and asymptotic confidence interval. Finally, a pair of real data sets is analyzed for illustrative purposes.
\end{abstract}

Keywords. Stress-strength model; Type-I progressive Hybrid censoring; Bayes estimator; Maximum likelihood estimator; Monte Carlo simulation.

MSC 2010: 62N01; 62N02.

\section{Introduction}

Hybrid censoring scheme that is a combination of the usual Type-I and TypeII censoring scenarios has been discussed extensively in the literature. Kundu

Corresponding author 
and Joarder (2006) proposed Type-I progressive hybrid censoring procedure by introducing a stopping time $\min \left(X_{m: m: n}, T\right)$. This approach is based on progressively Type-II censored order statistics $X_{1: m: n} \leqslant \cdots \leqslant X_{m: m: n}$, and it guarantees that the life test would not last beyond time $T$. $T$ is prefixed time and is named threshold time. Suppose $X_{1: m: n}, \ldots, X_{m: m: n}$ are the progressively Type-II right censored order statistics corresponding to $n$ units on a life-test with the progressive censoring scheme $\boldsymbol{r}=\left(r_{1}, \ldots, r_{m}\right)$. Then, the Type-I progressive hybrid censoring arises if the termination time of the life-test is chosen to be $\min \left(X_{m: m: n}, T\right)$. We denote this censoring scheme by $(\boldsymbol{r}, T)$. Under this censoring scheme, we have one of the two following types of observations:

$$
\begin{aligned}
\text { Case I : } & \left\{X_{1: m: n} \leqslant \cdots \leqslant X_{m: m: n}\right\} \quad \text { if } \quad X_{m: m: n} \leqslant T \\
\text { Case II : } & \left\{X_{1: m: n} \leqslant \cdots \leqslant X_{d: m: n}\right\} \quad \text { if } \quad d<m, \quad X_{d: m: n}<T<X_{d: m: n},
\end{aligned}
$$

where $X_{1: m: n} \leqslant X_{2: m: n} \leqslant \ldots$ denote the observed ordered failure times of the experimental units and $d$ is the number of failures before the time $T$, where $d$ is a random variable with support $\{0,1, \ldots, m\}$.

In the stress-strength modelling, $R=P(Y<X)$ is a measure of component reliability when it is subjected to random stress $Y$ and has strength $X$. It has many applications in engineering and medical sciences. For more details and applications, see Kotz et al. (2003). Much work has been done on the problem of estimating $R$ of some distributions. Such as, Kundu and Gupta (2005), Kundu and Gupta (2006), Saracoglu et al. (2012), Asgharzadeh et al. (2011; 2013), Asgharzadeh and Kazemi (2014), Govidarajulu (1967), Enis and Geisser (1971), Downtown (1973), Awad et al. (1981), Sathe and Shah (1981), Awad and Gharraf (1986), Constantine et al. (1986), Gupta and Gupta (1990), McCool (1991), Nandi and Aich (1996), Surles and Padgett (1998), Gupta et al. (1999), Gupta and Brown (2001), Raqab and Kundu (2005), Mokhlis (2005), Raqab et al. (2008), Kundu and Raqab (2009), Gupta et al. (2010), Rao et al. (2013) and Rao et al. (2016).

The exponential distribution is a simple and fundamental lifetime model and is a member of such important families of reliability distributions as Weibull and gamma; see Balakrishnan and Basu (1995). The exponential distribution denoted by $\operatorname{Exp}(\theta)$ has the probability density function (pdf)

$$
f(x ; \theta)=\frac{1}{\theta} e^{-\frac{x}{\theta}}, \quad x>0, \theta>0,
$$


and the cumulative distribution function (cdf)

$$
F(x ; \theta)=1-e^{-\frac{x}{\theta}}, \quad x>0, \theta>0 .
$$

Here $\theta$ is a scale parameter.

Based on Type-I progressively hybrid censored samples, we consider the estimation of $R=P(Y<X)$ when $X$ and $Y$ have two independent exponential distributions with different parameters.

\section{Maximum Likelihood Estimator of $R$}

Let $X \sim \operatorname{Exp}\left(\theta_{1}\right)$ and $Y \sim \operatorname{Exp}\left(\theta_{2}\right)$ be independent random variables. Then it can be easily seen that

$$
R=P(Y<X)=\frac{\theta_{1}}{\theta_{1}+\theta_{2}} .
$$

Our interest is estimating $R$ based on Type-I progressively hybrid censored data on both variables. To derive the MLE of $R$, first we obtain the MLEs of $\theta_{1}$ and $\theta_{2}$. Suppose $\boldsymbol{X}=\left(X_{1: m_{1}: n_{1}}, \ldots, X_{d_{1}: m_{1}: n_{1}}\right)$ is a Type-I progressively hybrid censored sample from $\operatorname{Exp}\left(\theta_{1}\right)$ with censored scheme $\left(\boldsymbol{r}_{1}, T_{1}\right)$ and $\boldsymbol{Y}=\left(Y_{1: m_{2}: n_{2}}, \ldots, Y_{d_{2}: m_{2}: n_{2}}\right)$ is a Type-I progressively hybrid censored sample from $\operatorname{Exp}\left(\theta_{2}\right)$ with censored scheme $\left(\boldsymbol{r}_{2}, T_{2}\right)$, where $\boldsymbol{r}_{i}=\left(r_{i 1}, r_{i 2}, \ldots, r_{i m_{i}}\right)$ and $\sum_{j=1}^{m_{i}} r_{i j}=n_{i}$, for $i=1,2$. For convenience, we will write $\left(X_{1}, \ldots, X_{d_{1}}\right)$ instead of $\left(X_{1: m_{1}: n_{1}}, \ldots, X_{d_{1}: m_{1}: n_{1}}\right)$, and $\left(Y_{1}, \ldots, Y_{d_{2}}\right)$ instead of $\left(Y_{1: m_{1}: n_{1}}, \ldots, Y_{d_{2}: m_{2}: n_{2}}\right)$. According to Cramér and Balakrishnan (2013), we have

$$
\begin{aligned}
L\left(\theta_{1}, \theta_{2}\right)= & c_{1} c_{2} \theta_{1}^{-d_{1}} \theta_{2}^{-d_{2}} \\
& \times \exp \left\{-\frac{1}{\theta_{1}}\left[\sum_{j=1}^{d_{1}}\left(1+r_{1 j}\right) X_{j}+\gamma_{d_{1}+1} T_{1}\right]\right. \\
& \left.-\frac{1}{\theta_{2}}\left[\sum_{j=1}^{d_{2}}\left(1+r_{2 j}\right) Y_{j}+\gamma_{d_{2}+1}^{\prime} T_{2}\right]\right\}
\end{aligned}
$$


where $c_{1}=\prod_{j=1}^{d_{1}} \gamma_{j}$ and $c_{2}=\prod_{j=1}^{d_{2}} \gamma_{j}^{\prime}$, such that $\gamma_{j}=\sum_{k=j}^{m_{1}}\left(1+r_{1 j}\right)$ and $\gamma_{j}^{\prime}=$ $\sum_{k=j}^{m_{2}}\left(1+r_{2 j}\right)$.

From (4), the log-likelihood function for the Type-I progressively hybrid censored data without the normalizing constant is

$$
\begin{aligned}
l\left(\theta_{1}, \theta_{2}\right)= & -d_{1} \log \left(\theta_{1}\right)-d_{2} \log \left(\theta_{2}\right)-\frac{1}{\theta_{1}}\left[\sum_{j=1}^{d_{1}}\left(1+r_{1 j}\right) X_{j}+\gamma_{d_{1}+1} T_{1}\right] \\
& -\frac{1}{\theta_{2}}\left[\sum_{j=1}^{d_{2}}\left(1+r_{2 j}\right) Y_{j}+\gamma_{d_{2}+1}^{\prime} T_{2}\right] .
\end{aligned}
$$

We denoted the MLEs of $\theta_{1}$ and $\theta_{2}$ by $\hat{\theta_{1}}$ and $\hat{\theta_{2}}$ respectively, where can be obtained as the solution of

$$
\begin{gathered}
\frac{\partial l}{\partial \theta_{1}}=-\frac{d_{1}}{\theta_{1}}+\frac{1}{\theta_{1}^{2}}\left[\sum_{j=1}^{d_{1}}\left(1+r_{1 j}\right) X_{j}+\gamma_{d_{1}+1} T_{1}\right]=0, \\
\frac{\partial l}{\partial \theta_{2}}=-\frac{d_{2}}{\theta_{2}}+\frac{1}{\theta_{2}^{2}}\left[\sum_{j=1}^{d_{2}}\left(1+r_{2 j}\right) Y_{j}+\gamma_{d_{2}+1}^{\prime} T_{2}\right]=0 .
\end{gathered}
$$

If $d_{1}>0$ and $d_{2}>0$, from (6) and (7), we obtain

$$
\begin{aligned}
& \hat{\theta}_{1}=\frac{1}{d_{1}}\left[\sum_{j=1}^{d_{1}}\left(1+r_{1 j}\right) X_{j}+\gamma_{d_{1}+1} T_{1}\right], \\
& \hat{\theta}_{2}=\frac{1}{d_{2}}\left[\sum_{j=1}^{d_{2}}\left(1+r_{2 j}\right) Y_{j}+\gamma_{d_{2}+1}^{\prime} T_{2}\right] .
\end{aligned}
$$

Therefore, we compute the MLE of $R$ as 


$$
\hat{R}=\frac{\hat{\theta}_{1}}{\hat{\theta}_{1}+\hat{\theta}_{2}} .
$$

But using the likelihood function (4), if $d_{1}=0$ or $d_{2}=0$, then $\hat{\theta}_{1}$ or $\hat{\theta}_{2}$ does not exist and therefore $\hat{R}$ does not exist.

\section{Asymptotic Confidence Interval of $R$}

In this section, we propose the asymptotic confidence interval of $R$, through computing the inverse of the observed Fisher information matrix $I\left(\theta_{1}, \theta_{2}\right)$ and using the delta method.

From the log-likelihood function in (5), we obtain the observed Fisher information matrix of $\left(\theta_{1}, \theta_{2}\right)$ as

$$
I\left(\theta_{1}, \theta_{2}\right)=-\left(\begin{array}{cc}
\frac{\partial^{2} l}{\partial \theta_{1}^{2}} & \frac{\partial^{2} l}{\partial \theta_{1} \partial \theta_{2}} \\
& \\
\frac{\partial^{2} l}{\partial \theta_{1} \partial \theta_{2}} & \frac{\partial^{2} l}{\partial \theta_{2}^{2}}
\end{array}\right)=\left(\begin{array}{cc}
I_{11} & I_{12} \\
I_{21} & I_{22}
\end{array}\right),
$$

where

$$
\begin{aligned}
-I_{11} & =\frac{d_{1}}{\theta_{1}^{2}}-\frac{2}{\theta_{1}^{3}}\left[\sum_{j=1}^{d_{1}}\left(1+r_{1 j}\right) X_{j}+\gamma_{d_{1}+1} T_{1}\right], \\
-I_{22} & =\frac{d_{2}}{\theta_{2}^{2}}-\frac{2}{\theta_{2}^{3}}\left[\sum_{j=1}^{d_{2}}\left(1+r_{2 j}\right) Y_{j}+\gamma_{d_{2}+1} T_{2}\right], \\
I_{12} & =I_{21}=0 .
\end{aligned}
$$

Now, the asymptotic variance-covariance matrix, $A=\left[a_{i j}\right]$, for the MLEs is obtained by inverting the Fisher information matrix as

$$
A=I^{-1}\left(\theta_{1}, \theta_{2}\right)=\frac{1}{I_{11} I_{22}}\left(\begin{array}{cc}
I_{22} & 0 \\
0 & I_{11}
\end{array}\right)=\left(\begin{array}{cc}
I_{11}^{-1} & 0 \\
0 & I_{22}^{-1}
\end{array}\right) .
$$

Now, the variance of $\hat{R}$, denoted by $B$, can be obtained use the delta method. 
We have $\hat{R}=g\left(\hat{\theta_{1}}, \hat{\theta_{2}}\right)$, where

$$
g\left(\theta_{1}, \theta_{2}\right)=\frac{\theta_{1}}{\theta_{1}+\theta_{2}}
$$

Therefore, $B=\boldsymbol{b}^{t} A \boldsymbol{b}$, where

$$
\boldsymbol{b}=\left(\begin{array}{c}
\frac{\partial g}{\partial \theta_{1}} \\
\frac{\partial g}{\partial \theta_{2}}
\end{array}\right)=\frac{1}{\left(\theta_{1}+\theta_{2}\right)^{2}}\left(\begin{array}{c}
\theta_{2} \\
-\theta_{1}
\end{array}\right) .
$$

It can be easily verified that

$$
B=\boldsymbol{b}^{t} A \boldsymbol{b}=\frac{1}{\left(\theta_{1}+\theta_{2}\right)^{4}}\left[\theta_{2}^{2} I_{11}^{-1}+\theta_{1}^{2} I_{22}^{-1}\right] .
$$

To compute the confidence interval of $R$, it is enough to estimate $B$. We can using the MLEs of $\theta_{1}$ and $\theta_{2}$, to estimate $B$. It can be easily verified that

$$
\hat{B}=\frac{d_{1}+d_{2}}{d_{1} d_{2}} \cdot \frac{\hat{\theta}_{1}^{2} \hat{\theta}_{2}^{2}}{\left(\hat{\theta}_{1}^{2}+\hat{\theta}_{2}^{2}\right)^{4}} .
$$

As a consequence, a $100(1-\alpha) \%$ asymptotic confidence interval of $R$ is

$$
\left(\hat{R}-Z_{1-\alpha / 2} \sqrt{\hat{B}}, \hat{R}+Z_{1-\alpha / 2} \sqrt{\hat{B}}\right),
$$

where $Z_{\alpha}$ is $100 \alpha$-th percentile of standard normal distribution.

\section{Bayes Estimator of $R$}

In this section, we obtain the Bayes estimator of $R$ under Type-I progressive hybrid censoring. For this purpose, we have the following theorem.

Theorem 1. Let $\lambda_{1}=\frac{1}{\theta_{1}} \sim \operatorname{Gamma}\left(a_{1}, b_{1}\right)$ and $\lambda_{2}=\frac{1}{\theta_{2}} \sim \operatorname{Gamma}\left(a_{2}, b_{2}\right)$ are independent, where $a_{1}$ and $a_{2}$ are the shape parameters and $b_{1}$ and $b_{2}$ are the rate parameters. Under the squared error loss function, the Bayes estimation of $R$ denoted by $\hat{R}_{B}$ as

$$
\hat{R}_{B}=\frac{a_{2}+d_{2}}{s}\left(\frac{b_{2}+w_{2}}{b_{1}+w_{1}}\right)^{a_{2}+d_{2}} F_{2,1}\left(s, a_{2}+d_{2}+1, s+1,1-\frac{b_{2}+w_{2}}{b_{1}+w_{1}}\right)
$$


such that

$$
\begin{aligned}
s & =a_{1}+d_{1}+a_{2}+d_{2}, \\
w_{1} & =\sum_{j=1}^{d_{1}}\left(1+r_{1 j}\right) x_{j}+\gamma_{d_{1}+1} T_{1}, \\
w_{2} & =\sum_{j=1}^{d_{2}}\left(1+r_{2 j}\right) y_{j}+\gamma_{d_{2}+1}^{\prime} T_{2},
\end{aligned}
$$

and $F_{2,1}$ is the hypergeometric function given by (see Saracoglu et al. (2012));

$$
\begin{aligned}
F_{2,1}(a, b, c, z) & =\frac{\Gamma(c)}{\Gamma(b) \Gamma(c-b)} \int_{0}^{1} \frac{t^{b-1}(1-t)^{c-b-1}}{(1-z t)^{a}} d t \\
& =\sum_{k=0}^{\infty} \frac{\Gamma(c) \Gamma(a+k) \Gamma(b+k) z^{k}}{\Gamma(a) \Gamma(b) \Gamma(c+k) k !} .
\end{aligned}
$$

Proof. To obtain the posterior distributions of $\lambda_{1}$ and $\lambda_{2}$, we have

$$
\begin{aligned}
& \pi\left(\lambda_{1} \mid \text { data }\right) \propto \lambda_{1}^{a_{1}+d_{1}-1} e^{-\left(b_{1}+w_{1}\right) \lambda_{1}}, \\
& \pi\left(\lambda_{2} \mid \text { data }\right) \propto \lambda_{2}^{a_{2}+d_{2}-1} e^{-\left(b_{2}+w_{2}\right) \lambda_{2}} .
\end{aligned}
$$

Consequently

$$
\begin{aligned}
\lambda_{1} \mid \text { data } & \sim \operatorname{Gamma}\left(a_{1}+d_{1}, b_{1}+w_{1}\right), \\
\lambda_{2} \mid \text { data } & \sim \operatorname{Gamma}\left(a_{2}+d_{2}, b_{2}+w_{2}\right) .
\end{aligned}
$$

Using the independency of $\lambda_{1}$ and $\lambda_{2}$, when $\lambda_{1}>0$ and $0<r<1$, we compute the joint pdf of $R=\frac{\lambda_{2}}{\lambda_{1}+\lambda_{2}}$ and $\lambda_{1}$ given the data as follows:

$$
\begin{aligned}
f_{\left(R, \lambda_{1}\right) \mid \text { data }}\left(r, \lambda_{1}\right)= & \frac{\lambda_{1}}{(1-r)^{2}} f_{\left(\lambda_{2}, \lambda_{1}\right) \mid \text { data }}\left(\frac{r \lambda_{1}}{1-r}, \lambda_{1}\right) \\
= & \frac{\lambda_{1}}{(1-r)^{2}} \cdot \frac{\left(b_{2}+w_{2}\right)^{a_{2}+d_{2}}}{\Gamma\left(a_{2}+d_{2}\right)}\left(\frac{r \lambda_{1}}{1-r}\right)^{a_{2}+d_{2}-1} e^{-\left(b_{2}+w_{2}\right) \frac{r \lambda_{1}}{1-r}} \\
& \times \frac{\left(b_{1}+w_{1}\right)^{a_{1}+d_{1}}}{\Gamma\left(a_{1}+d_{1}\right)} \lambda_{1}^{a_{1}+d_{1}-1} e^{-\left(b_{1}+w_{1}\right) \lambda_{1}}
\end{aligned}
$$




$$
\begin{aligned}
= & \frac{\left(b_{1}+w_{1}\right)^{a_{1}+d_{1}}}{\Gamma\left(a_{1}+d_{1}\right)} \cdot \frac{\left(b_{2}+w_{2}\right)^{a_{2}+d_{2}}}{\Gamma\left(a_{2}+d_{2}\right)} \cdot \frac{r^{a_{2}+d_{2}-1}}{(1-r)^{a_{2}+d_{2}+1}} \\
& \times \lambda_{1}^{s-1} e^{-\left[b_{1}+w_{1}+\frac{r}{1-r}\left(b_{2}+w_{2}\right)\right] \lambda_{1}} .
\end{aligned}
$$

Now, we can obtain pdf of $R$ given the data by integrating with respect to $\lambda_{1}$. So for $0<r<1$,

$$
\begin{aligned}
f_{R \mid \text { data }}(r)= & \frac{\left(b_{1}+w_{1}\right)^{a_{1}+d_{1}}}{\Gamma\left(a_{1}+d_{1}\right)} \cdot \frac{\left(b_{2}+w_{2}\right)^{a_{2}+d_{2}}}{\Gamma\left(a_{2}+d_{2}\right)} \cdot \frac{r^{a_{2}+d_{2}-1}}{(1-r)^{a_{2}+d_{2}+1}} \\
& \times \int_{0}^{\infty} \lambda_{1}^{s-1} e^{-\left[b_{1}+w_{1}+\frac{r}{1-r}\left(b_{2}+w_{2}\right)\right] \lambda_{1}} d \lambda_{1} \\
= & \frac{\left(b_{1}+w_{1}\right)^{a_{1}+d_{1}}}{\Gamma\left(a_{1}+d_{1}\right)} \cdot \frac{\left(b_{2}+w_{2}\right)^{a_{2}+d_{2}}}{\Gamma\left(a_{2}+d_{2}\right)} \cdot \frac{r^{a_{2}+d_{2}-1}}{(1-r)^{a_{2}+d_{2}+1}} \\
& \times \frac{\Gamma(s)}{\left(b_{1}+w_{1}+\frac{r}{1-r}\left(b_{2}+w_{2}\right)\right)^{s}} \\
= & \frac{\left(b_{1}+w_{1}\right)^{a_{1}+d_{1}}}{\Gamma\left(a_{1}+d_{1}\right)} \cdot \frac{\left(b_{2}+w_{2}\right)^{a_{2}+d_{2}}}{\Gamma\left(a_{2}+d_{2}\right)} \Gamma(s) \\
& \times \frac{r^{a_{2}+d_{2}-1}(1-r)^{a_{1}+d_{1}-1}}{\left((1-r)\left(b_{1}+w_{1}\right)+r\left(b_{2}+w_{2}\right)\right)^{s}} .
\end{aligned}
$$

Now, under the squared error loss function, we obtain $\hat{R}_{B}$ as follows:

$$
\begin{aligned}
\hat{R}_{B}= & \int_{0}^{1} r f_{R \mid \text { data }}(r) d r \\
= & \frac{\left(b_{1}+w_{1}\right)^{a_{1}+d_{1}}}{\Gamma\left(a_{1}+d_{1}\right)} \cdot \frac{\left(b_{2}+w_{2}\right)^{a_{2}+d_{2}}}{\Gamma\left(a_{2}+d_{2}\right)} \Gamma(s) \\
& \times \int_{0}^{1} \frac{r^{a_{2}+d_{2}}(1-r)^{a_{1}+d_{1}-1}}{\left((1-r)\left(b_{1}+w_{1}\right)+r\left(b_{2}+w_{2}\right)\right)^{s}} d r \\
= & \frac{\left(b_{1}+w_{1}\right)^{a_{1}+d_{1}}}{\Gamma\left(a_{1}+d_{1}\right)} \cdot \frac{\left(b_{2}+w_{2}\right)^{a_{2}+d_{2}}}{\Gamma\left(a_{2}+d_{2}\right)} \cdot \frac{\Gamma(s)}{\left(b_{1}+w_{1}\right)^{s}}
\end{aligned}
$$




$$
\begin{aligned}
& \times \int_{0}^{1} \frac{r^{a_{2}+d_{2}}(1-r)^{a_{1}+d_{1}-1}}{\left(1-\frac{b_{1}+w_{1}-\left(b_{2}+w_{2}\right)}{b_{1}+w_{1}} r\right)^{s}} d r \\
= & \frac{\left(b_{1}+w_{1}\right)^{a_{1}+d_{1}}}{\Gamma\left(a_{1}+d_{1}\right)} \cdot \frac{\left(b_{2}+w_{2}\right)^{a_{2}+d_{2}}}{\Gamma\left(a_{2}+d_{2}\right)} \cdot \frac{\Gamma(s)}{\left(b_{1}+w_{1}\right)^{s}} \\
& \times \frac{\Gamma\left(a_{2}+d_{2}+1\right) \Gamma\left(a_{1}+d_{1}\right)}{\Gamma(s+1)} \\
& \times F_{2,1}\left(s, a_{2}+d_{2}+1, s+1,1-\frac{b_{2}+w_{2}}{b_{1}+w_{1}}\right) \\
= & \frac{a_{2}+d_{2}}{s}\left(\frac{b_{2}+w_{2}}{b_{1}+w_{1}}\right)^{a_{2}+d_{2}} \\
& \times F_{2,1}\left(s, a_{2}+d_{2}+1, s+1,1-\frac{b_{2}+w_{2}}{b_{1}+w_{1}}\right) .
\end{aligned}
$$

\section{Simulation Study}

In this section, Monte Carlo simulations are carried out to evaluate the performance of the MLEs, Bayes estimators and asymptotic confidence intervals for different censoring schemes. The computations are performed using $\mathrm{R}$ software and Mathematica software. We mainly evaluate the performances of the MLEs and Bayes estimators in terms of average estimators and mean of square errors (MSE). Also we evaluate the performances of the asymptotic confidence intervals in terms of average lengths and coverage probabilities.

From the sample, we compute the MLE and Bayes estimator of $R$ using (10) and (11), respectively. We compute the MLE and Bayes estimator of $R$ for different Type-I progressive hybrid censoring schemes $\left(\boldsymbol{r}_{1}, T_{1}\right)$ and $\left(\boldsymbol{r}_{2}, T_{2}\right)$, and we report the average estimator and MSE of the MLEs and Bayes estimators of $R$ by 20,000 replications. The results are represented in the Table 2. The Type-II progressive censoring schemes that are employed in computations, have represented in the Table 1. Also the priors are as follows:

$$
\begin{aligned}
\text { Prior I : } & a_{1}=0, b_{1}=0, a_{2}=0, b_{2}=0, \\
\text { Prior II : } & a_{1}=2, b_{1}=1, a_{2}=3, b_{2}=5, \\
\text { Prior III : } & a_{1}=0.3, b_{1}=1, a_{2}=0.7, b_{2}=5,
\end{aligned}
$$


Table 1. Type-II progressive censoring schemes.

\begin{tabular}{cccc}
\hline \hline$n$ & $m$ & $\boldsymbol{r}$ & scheme number \\
\hline 30 & 5 & $(5,5,5,5,5)$ & {$[1]$} \\
& 10 & $(4,0,4,0, \ldots, 4,0)$ & {$[2]$} \\
& 15 & $(15,0,0, \ldots, 0)$ & {$[3]$} \\
& 20 & $(0, \ldots, 0,10)$ & {$[4]$} \\
40 & 5 & $(7,7,7,7,7)$ & {$[5]$} \\
& 10 & $(6,0,6,0, \ldots, 6,0)$ & {$[6]$} \\
& 15 & $(25,0, \ldots, 0)$ & {$[7]$} \\
& 20 & $(0, \ldots, 0,20)$ & {$[8]$} \\
\hline
\end{tabular}

where the Prior I is a non-informative gamma prior and the Priors II and III are informative gamma priors.

From Table 2, in the most considered cases, we observe that for fixed $n_{1}$ and $n_{2}$, as $m_{1}, m_{2}, T_{1}$ or $T_{2}$ increases, the MSE decreases. Also we observe that the results of MLEs and Bayes estimators under Prior I are so similar, that is reasonable, since Prior I is a non-informative prior. Furthermore Bayes estimators under Prior II and Prior III are better than the MLEs and Bayes estimators under Prior I, that is reasonable too, since the priors II and III are informative priors.

Also we obtain $95 \%$ asymptotic confidence intervals of $R$ by simulating 20,000 samples under different Type-I progressive hybrid censoring schemes, and obtain the average lengths and coverage probabilities of asymptotic confidence intervals. The results are represented in the Table 3.

From Table 3, in the all considered cases, we observe that coverage probabilities are almost equal and less than 0.95 , where this is reasonable, since the variance of $\hat{R}$ is estimated using one sample alone. Also in all situations, for fixed $n_{1}$ and $n_{2}$, as $m_{1}, m_{2}, T_{1}$ or $T_{2}$ increases, the average length decreases. This is reasonable too.

\section{Data Analysis}

In this section, the analysis of a pair of real data sets is presented for illustrative purposes. Tables 4 and 5 show the breaking strengths of jute fiber at two different gauge lengths. These two data sets were used by Xia et al. (2009). Both data sets in Tables 4 and 5 have the exponential distribution 
Table 2. The average estimators (A.E) and MSE of the MLEs and Bayes estimators of $R$ when $\left(\theta_{1}, \theta_{2}\right)=(1,2)$.

\begin{tabular}{|c|c|c|c|c|c|c|}
\hline \multirow[t]{2}{*}{$\left(\boldsymbol{r}_{1}, T_{1}\right)$} & \multirow[t]{2}{*}{$\left(\boldsymbol{r}_{2}, T_{2}\right)$} & & \multirow[t]{2}{*}{ MLE } & \multicolumn{3}{|c|}{ Bayes } \\
\hline & & & & Prior I & Prior II & Prior III \\
\hline \multirow{8}{*}{$([1], 1)$} & $([5], 1)$ & A.E & 0.34505 & 0.35667 & 0.32606 & 0.31695 \\
\hline & & MSE & 0.01954 & 0.01793 & 0.00904 & 0.00893 \\
\hline & $([6], 1)$ & A.E & 0.32929 & 0.34646 & 0.32157 & 0.33553 \\
\hline & & MSE & 0.01478 & 0.01433 & 0.00852 & 0.00941 \\
\hline & $([7], 2)$ & A.E & 0.32284 & 0.34168 & 0.31659 & 0.33692 \\
\hline & & MSE & 0.01371 & 0.01328 & 0.00822 & 0.00944 \\
\hline & $([8], 2)$ & A.E & 0.32717 & 0.34982 & 0.31924 & 0.36278 \\
\hline & & MSE & 0.01165 & 0.01142 & 0.00764 & 0.00888 \\
\hline \multirow{8}{*}{$([2], 1)$} & $([5], 1)$ & A.E & 0.35541 & 0.35379 & 0.33847 & 0.30148 \\
\hline & & MSE & 0.01715 & 0.01505 & 0.00768 & 0.00803 \\
\hline & $([6], 1)$ & A.E & 0.33905 & 0.34402 & 0.33119 & 0.32056 \\
\hline & & MSE & 0.01158 & 0.01121 & 0.00712 & 0.00760 \\
\hline & $([7], 2)$ & A.E & 0.33069 & 0.33881 & 0.32709 & 0.32098 \\
\hline & & MSE & 0.01014 & 0.00969 & 0.00674 & 0.00777 \\
\hline & $([8], 2)$ & A.E & 0.33593 & 0.34703 & 0.32813 & 0.34329 \\
\hline & & MSE & 0.00790 & 0.00784 & 0.00579 & 0.00630 \\
\hline \multirow{8}{*}{$([3], 1.5)$} & $([5], 1)$ & A.E & 0.36232 & 0.35786 & 0.34425 & 0.30165 \\
\hline & & MSE & 0.01637 & 0.01489 & 0.00714 & 0.00772 \\
\hline & $([6], 1)$ & A.E & 0.34488 & 0.34759 & 0.33683 & 0.31762 \\
\hline & & MSE & 0.01067 & 0.01030 & 0.00669 & 0.00713 \\
\hline & $([7], 2)$ & A.E & 0.33740 & 0.34160 & 0.33374 & 0.32108 \\
\hline & & MSE & 0.00921 & 0.00908 & 0.00616 & 0.00734 \\
\hline & $([8], 2)$ & A.E & 0.34228 & 0.34761 & 0.33690 & 0.34354 \\
\hline & & MSE & 0.00695 & 0.00712 & 0.00532 & 0.00578 \\
\hline \multirow{8}{*}{$([4], 1.5)$} & $([5], 1)$ & A.E & 0.35738 & 0.34880 & 0.34312 & 0.28544 \\
\hline & & MSE & 0.01433 & 0.01253 & 0.00601 & 0.00744 \\
\hline & $([6], 1)$ & A.E & 0.33987 & 0.33800 & 0.33671 & 0.30400 \\
\hline & & MSE & 0.00885 & 0.00831 & 0.00548 & 0.00625 \\
\hline & $([7], 2)$ & A.E & 0.33289 & 0.33258 & 0.33185 & 0.30543 \\
\hline & & MSE & 0.00756 & 0.00712 & 0.00505 & 0.00615 \\
\hline & $([8], 2)$ & A.E & 0.33786 & 0.33910 & 0.33914 & 0.33353 \\
\hline & & MSE & 0.00508 & 0.00484 & 0.00424 & 0.00424 \\
\hline
\end{tabular}


Table 3. The average lengths (A.L) and coverage probabilities (CP) of asymptotic confidence intervals of $R$ when $\left(\theta_{1}, \theta_{2}\right)=(1,2)$.

\begin{tabular}{|c|c|c|c|}
\hline$\left(\boldsymbol{r}_{1}, T_{1}\right)$ & $\left(\boldsymbol{r}_{2}, T_{2}\right)$ & & CI \\
\hline \multirow{8}{*}{$([1], 1)$} & $([5], 1)$ & A. $\mathrm{L}$ & 0.51428 \\
\hline & & $\mathrm{CP}$ & 0.88000 \\
\hline & $([6], 1)$ & A. $\mathrm{L}$ & 0.45777 \\
\hline & & $\mathrm{CP}$ & 0.88290 \\
\hline & $([7], 2)$ & A. $\mathrm{L}$ & 0.44387 \\
\hline & & $\mathrm{CP}$ & 0.88555 \\
\hline & $([8], 2)$ & A. $\mathrm{L}$ & 0.40900 \\
\hline & & $\mathrm{CP}$ & 0.89410 \\
\hline \multirow{8}{*}{$([2], 1)$} & $([5], 1)$ & A.L & 0.46572 \\
\hline & & $\mathrm{CP}$ & 0.89890 \\
\hline & $([6], 1)$ & A. $\mathrm{L}$ & 0.40160 \\
\hline & & $\mathrm{CP}$ & 0.91035 \\
\hline & $([7], 2)$ & A. $\mathrm{L}$ & 0.38451 \\
\hline & & $\mathrm{CP}$ & 0.91125 \\
\hline & $([8], 2)$ & A. $L$ & 0.33854 \\
\hline & & $\mathrm{CP}$ & 0.91715 \\
\hline \multirow{8}{*}{$([3], 1.5)$} & $([5], 1)$ & A.L & 0.45436 \\
\hline & & $\mathrm{CP}$ & 0.90575 \\
\hline & $([6], 1)$ & A. $\mathrm{L}$ & 0.38475 \\
\hline & & $\mathrm{CP}$ & 0.91445 \\
\hline & $([7], 2)$ & A.L & 0.36776 \\
\hline & & $\mathrm{CP}$ & 0.92275 \\
\hline & $([8], 2)$ & A. $\mathrm{L}$ & 0.31670 \\
\hline & & $\mathrm{CP}$ & 0.92740 \\
\hline \multirow{8}{*}{$([4], 1.5)$} & $([5], 1)$ & A.L & 0.42741 \\
\hline & & $\mathrm{CP}$ & 0.90805 \\
\hline & $([6], 1)$ & A. $L$ & 0.34971 \\
\hline & & $\mathrm{CP}$ & 0.92280 \\
\hline & $([7], 2)$ & A. $L$ & 0.33015 \\
\hline & & $\mathrm{CP}$ & 0.92655 \\
\hline & $([8], 2)$ & A. $L$ & 0.27147 \\
\hline & & $\mathrm{CP}$ & 0.92875 \\
\hline
\end{tabular}


Table 4. Data Set 1 (Breaking strength of jute fiber of gauge length $10 \mathrm{~mm}$ ).

\begin{tabular}{cccccccccc}
\hline \hline 693.73 & 704.66 & 323.83 & 778.17 & 123.06 & 637.66 & 383.43 & 151.48 & 108.94 & 50.16 \\
671.49 & 183.16 & 257.44 & 727.23 & 291.27 & 101.15 & 376.42 & 163.40 & 141.38 & 700.74 \\
262.90 & 353.24 & 422.11 & 43.93 & 590.48 & 212.13 & 303.90 & 506.60 & 530.55 & 177.25 \\
\hline
\end{tabular}

Table 5. Data Set 2 (Breaking strength of jute fiber of gauge length $20 \mathrm{~mm}$ ).

\begin{tabular}{cccccccccc}
\hline \hline 71.46 & 419.02 & 284.64 & 585.57 & 456.60 & 113.85 & 187.85 & 688.16 & 662.66 & 45.58 \\
578.62 & 756.70 & 594.29 & 166.49 & 99.72 & 707.36 & 765.14 & 187.13 & 145.96 & 350.70 \\
547.44 & 116.99 & 375.81 & 581.60 & 119.86 & 48.01 & 200.16 & 36.75 & 244.53 & 83.55 \\
\hline
\end{tabular}

and based on the complete data set, $\hat{R}=0.51768$. See Asgharzadeh and Kazemi (2014).

For illustrative purposes, we have considered two different Type-I progressive hybrid censoring schemes.

\section{Scheme 1:}

$$
\begin{array}{ll}
\boldsymbol{r}_{1}=(1,1,1,1,1,1,1,1,1,1,1,1,1,1,1), & T_{1}=300 \\
\boldsymbol{r}_{2}=(1,3,4,1,1,3,1,2,2,2), & T_{2}=200 .
\end{array}
$$

Applying these censoring scheme, we have obtained

$$
\begin{aligned}
\boldsymbol{x}= & (43.93,50.16,101.15,108.94,123.06,141.38,151.48,177.25 \\
& , 183.16,212.13,257.4) \\
\boldsymbol{y}= & (36.75,45.58,48.01,71.46,99.72,113.85,119.86,116.49,187.85) .
\end{aligned}
$$

In this case, we have $\hat{R}=0.5847, \hat{R}_{B}^{(I)}=0.5897, \hat{R}_{B}^{(I I)}=0.6195, \hat{R}_{B}^{(I I I)}=$ 0.6012 , and $95 \%$ asymptotic confidence interval of $R$ is $(0.3708,0.7986)$.

\section{Scheme 2:}

$$
\begin{aligned}
& \boldsymbol{r}_{1}=\boldsymbol{r}_{2}=(5,5,5,5,5) \\
& T_{1}=T_{2}=150 .
\end{aligned}
$$


Applying these censoring scheme, we have obtained

$$
\begin{aligned}
& \boldsymbol{x}=(43.93,50.16,108.94), \\
& \boldsymbol{y}=(36.75,45.58,71.46,83.55) .
\end{aligned}
$$

In this case, we have $\hat{R}=0.5713, \hat{R}_{B}^{(I)}=0.6264, \hat{R}_{B}^{(I I)}=0.6402, \hat{R}_{B}^{(I I I)}=$ 0.6422 , and $95 \%$ asymptotic confidence interval for $R$ is $(0.2047,0.9379)$.

\section{Conclusion}

In this paper, we considered the estimation of $R=P(Y<X)$ based on Type-I progressive hybrid censored samples. It is assumed that $X$ and $Y$ are two independent exponential distributions with different parameters. We derived the maximum likelihood and Bayes estimators of $R$ and obtained the asymptotic confidence intervals of $R$. We evaluated performance of MLEs, Bayes estimators and asymptotic confidence intervals via simulation. Finally, we consider a pair of real data sets and computed the MLEs, Bayes estimators and asymptotic confidence intervals under two different Type-I progressive hybrid censoring schemes.

\section{Acknowledgement}

The authors are highly grateful to the editor and referees for their helpful and valuable comments and suggestions for improving the paper.

\section{References}

Asgharzadeh, A. and Kazemi, M. (2014). Stress-strength Reliability of Exponential Distribution based on Hybrid Censored Samples. Proceeding of 12th the Iranian Statistical Conference, 25-27 August, Razi University, Iran.

Asgharzadeh, A., Valiollahi, R., Raqab, M.Z. (2013). Estimation of the Stress-strength Reliability for the Generalized Logistic Distribution. Statistical Methodology, 15, 73-94.

Asgharzadeh, A., Valiollahi, R. and Raqab, M.Z. (2011). Stress-strength Reliability of Weibull Distribution based on Progressively Censored Samples. SORT, 35, 103-124.

Awad, A.M., Azzam, M.M. and Hamadan, M.A. (1981). Some Inference Results in $P(Y<X)$ in the Bivariate Exponential Model. Communications in Statistics - Theory and Methods, 20, 2515-2525. 
Awad, A.M. and Gharraf, M.K. (1986). Estimation of $P(Y<X)$ in the Burr Case: a Comparative Study. Communications in Statistics - Simulation and Computation, 15, 389403.

Balakrishnan, N. and Basu, A.P. (eds.) (1995). The Exponential Distributions: Theory, Methods, and Applications. Taylor and Francis, Newark, NJ.

Cramér, E. and Balakrishnan, N. (2013). On Some Exact Distributional Results based on Type-I Progressively Hybrid Censored Data from Exponential Distributions. Statistical Methodology, 10, 128-150.

Constantine, K., Tse, S.K. and Karson, M. (1986). Estimation of $P(Y<X)$ in Gamma Case. Communications in Statistics - Simulation and Computation, 15, 365-388.

Downtown, F. (1973). The Estimation of $P(X<Y)$ in the Normal Case. Technometrics, 15, 551-558.

Enis, P. and Geisser, S. (1971). Estimation of the Probability that $Y<X$. Journal of the American Statistical Association, 66(333), 162-168.

Govidarajulu, Z. (1967). Two Sided Confidence Limits for $P(X>Y)$ based on Normal Samples of $X$ and $Y$. Sankhya: The Indian Journal of Statistics, Series B, 29, 35-40.

Gupta, R.C. and Brown, N. (2001) Reliability Studies of the Skew-Normal Distribution and Its Application to Strength-stress Models. Communications in Statistics - Theory and Methods, 30, 2427-2445.

Gupta, R.C., Ghitany, M.E. and Al-Mutairib, D.K. (2010). Estimation of Reliability from Marshall-Olkin Extended Lomax Distributions. Journal of Statistical Computation and Simulation, 80, 937-947.

Gupta, R.D. and Gupta, R.C. (1990). Estimation of $P(a X>b Y)$ in the Multivariate Normal Case. Statistics, 21, 91-97.

Gupta, R.C., Ramakrishnan, S. and Zhou, X. (1999). Point and Interval Estimation of $\operatorname{Pr}(X>Y)$ : the Normal Case with Common Coefficient of Variation. Annals of the Institute of Statistical Mathematics, 51, 571-584.

Kotz, S., Lumelskii, Y. and Pensky, M. (2003). The Stress-Strength Model and its Generalizations. World Scientific, New York.

Kundu, D. and Gupta, R.D. (2005). Estimation of $P(Y<X)$ for the Generalized Exponential Distribution. Metrika, 61, 291-308.

Kundu, D. and Gupta, R.D. (2006). Estimation of $P(Y<X)$ for Weibull Distributions. IEEE Transcations on Reliability, 55, 270-280. 
Kundu, D. and Joarder, A. (2006). Analysis of Type-II Progressively Hybrid Censored Data. Computational Statistics and Data Analysis, 50, 2509-2528.

Kundu, D. and Raqab, M.Z. (2009). Estimation of $R=P(Y<X)$ for Three-parameter Weibull Distribution. Statistics and Probability Letters, 79, 1839-1846.

McCool, J.I. (1991). Inference on $P(Y<X)$ in the Weibull Case. Communications in Statistics - Simulation and Computation, 20, 129-148.

Mokhlis, N.A. (2005). Reliability of a Stress- strength Model with Burr Type III Distributions. Communications in Statistics - Theory and Methods, 34, 1643-1657.

Nandi, S.B. and Aich, A.B. (1996). A Note on Estimation of $P(X>Y)$ for Some Distributions Useful in Life-testing. IAPQR Transactions, 19, 35-44.

Rao, G.S., Kantam, R.R.L., Rosaiah, K. and Pratapa, J.R. (2013). Estimation of Stressstrength Reliability from Inverse Rayleigh Distribution. Journal of Industrial and Production Engineering, 30, 254-263.

Rao, G.S., Rosaiah, K. and Babu, M.S. (2016). Estimation of Stress-strength Reliability from Exponentiated Fréchet Distribution. The International Journal of Advanced Manufacturing Technology, DOI 10.1007/s00170-016-8404-z: 1-9.

Raqab, M.Z. and Kundu, D. (2005). Comparison of Different Estimators of $P(Y<X)$ for a Scaled Burr Type X Distribution. Communications in Statistics - Simulation and Computation, 34, 465-483.

Raqab, M.Z., Madi, M.T. and Kundu, D. (2008). Estimation of $P(Y<X)$ for the 3parameter Generalized Exponential Distribution. Communications in Statistics - Theory and Methods, 37, 2854-2864.

Saracoglu, B., Kinaci, I. and Kundu, D. (2012). On Estimation of $P(Y<X)$ for Exponential Distribution under Progressive Type-II Censoring. Journal of statistical computation and simulation, 82, 729-744.

Sathe, Y.S. and Shah, S.P. (1981). On Estimation $P(Y<X)$ for the Exponential Distribution. Communications in Statistics - Theory and Methods, 10, 39-47.

Surles, J.G. and Padgett, W.J. (1998). Inference for $P(Y<X)$ in the Burr Type X Model. Journal of Applied Statistical Science, 7, 225-238.

Tong, H. (1977). On the Estimation of $P(Y<X)$ for Exponential Families. IEEE Transactions on Reliability, 26, 54-56.

Xia, Z.P., Yu, J.Y., Cheng, L.D., Liu, L.F. and Wang, W.M. (2009). Study on the Breaking Strength of Jute Fibers Using Modified Weibull Distribution. Journal of Composites Part A: Applied Science and Manufacturing, 40, 54-59. 


\section{S. M. Mirjalili}

Department of Statistics,

Yazd University,

Yazd, Iran.

email:mirjalili8@yahoo.com

\section{H. Nadeb}

Department of Statistics,

Yazd University,

Yazd, Iran.

email: honadeb@yahoo.com

\section{H. Torabi}

Department of Statistics,

Yazd University,

Yazd, Iran.

email: htorabi@yazd.ac.ir

\section{S. F. Bafekri}

Department of Statistics,

Yazd University,

Yazd, Iran.

email:s.bafekri@gmail.com 